\title{
Apical barriers to airway epithelial cell gene transfer with amphotropic retroviral vectors
}

\author{
G Wang ${ }^{1,4}$, G Williams ${ }^{1}, \mathrm{H} \mathrm{Xia}^{1}$, M Hickey ${ }^{1}, \mathrm{~J} \mathrm{Shao}^{3}$, BL Davidson ${ }^{2}$ and PB McCray ${ }^{1}$ \\ ${ }^{1}$ Program in Gene Therapy, Department of Pediatrics, University of Iowa College of Medicine, Iowa City, IA, USA; ${ }^{2}$ Program in \\ Gene Therapy, Department of Internal Medicine, University of Iowa College of Medicine, Iowa City, IA, USA; and ${ }^{3}$ Central \\ Microscopy Research Facility, University of Iowa College of Medicine, Iowa City, IA, USA
}

\begin{abstract}
Gene transfer to airway epithelia with amphotropic pseudotyped retroviral vectors is inefficient following apical vector application. To better understand this inefficiency, we localized the expression of Pit2, the amphotropic receptor, in polarized human airway epithelia. Pit2 was expressed on both the apical and basolateral surfaces of the cells, suggesting that factors other than receptor abundance may limit apical gene transfer efficiency. Binding studies performed with radiolabeled amphotropic MuLV suggested that the apically applied virus binds to Pit2. Hypothetical barriers to retroviral gene transfer include the apical glycocalyx and other secreted products of epithelia. In this study, we demonstrated that sialic acid, keratan sulfate and collagen type $V$ are present on the apical surface of well-differentiated human airway epithelia. While enzyme treatment reduced
\end{abstract}

the abundance of these components, the treatment also decreased the transepithelial resistance to $\sim 35 \%$ of the controls, suggesting that the epithelial integrity was impaired. To attain an airway epithelial culture with a modified apical surface and intact epithelial integrity, we utilized $100 \mathrm{mM}$ 2deoxy-D-glucose, a glycosylation inhibitor, to prevent the glycocalyx from reforming following enzyme treatment. This approach allowed the resistance, but not the apical glycocalyx to recover. Despite this physical modification of the cell surface, the amphotropic retroviral vector failed to transduce airway epithelia following apical application. These results suggest that factors other than apical receptor abundance and the glycocalyx inhibit amphotropic retroviral gene transfer in human airway epithelia.

Gene Therapy (2002) 9, 922-931. doi:10.1038/sj.gt.3301714

Keywords: amphotropic; Pit2; retrovirus; glycocalyx; cystic fibrosis

\section{Introduction}

Gene transfer is a direct approach to treat inherited lung diseases, such as cystic fibrosis (CF). To correct or prevent the chronic lung disease associated with this autosomal recessive disease it is anticipated that some level of transgene expression must be continuously maintained. ${ }^{1}$ This could be achieved using integrating vectors, such as Moloney murine leukemia virus (MuLV) and lentivirusbased retroviral vectors ${ }^{2}$ or adeno-associated virus (AAV) vectors. ${ }^{3}$ The most practical route for the direct delivery of vectors to the airway epithelium is by aerosolization or instillation. However, several studies indicate that the efficiency of gene transfer to polarized airway epithelia following apical application of viral vectors is poor, while transduction from the basolateral side occurs much more readily. Examples include serotype 5 adenovirus vectors ${ }^{4,5}$

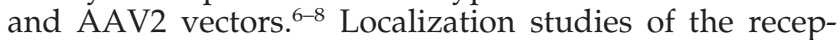
tors for adenovirus and AAV2 suggest that their expression is polarized to the basolateral surface, thus

Correspondence: PB McCray Jr, Department of Pediatrics, University of Iowa College of Medicine, Iowa City, IA 52242, USA

${ }^{4}$ Current address: Louisiana State University Health Sciences Center, Gene Therapy Program, Departments of Medicine and Genetics, 533 Bolivar St, CSB 642, New Orleans, LA 70112, USA

Received 26 September 2001; accepted 11 February 2002 offering a reasonable explanation for this apical inefficiency.

In well-differentiated human airway epithelia, we previously reported that $\mathrm{MuLV}$-based retrovirus or feline immunodeficiency virus (FIV)-based lentivirus vectors pseudotyped with the amphotropic, xenotropic, and VSV-G envelope glycoproteins do not transduce cells when applied apically. ${ }^{9-11}$ In contrast, the same envelopes infect epithelia readily when applied to the basolateral surface. The most simple intrepretation of these data is that the receptors for these envelopes are only expressed on the basolateral surface. However, there have been no studies localizing the expression of known retrovirus receptors in polarized airway epithelia. The receptor for the amphotropic pseudotyped retrovirus, Pit2, is an inorganic phosphate transporter, ${ }^{12}$ while the receptor for xenotropic enveloped retrovirus was identified as XPR1, a gene of unknown function. ${ }^{13-15}$ A putative receptor for the VSV-G envelope glycoprotein was suggested to be phosphatidylserine, but it is possible that other receptors or co-receptors exist. ${ }^{16}$ In the present study, we sought to better understand the limitations to apical gene transfer with retroviruses, using the amphotropic enveloped MuLV-based vector as a model.

Several steps are required for the successful transduction of a target cell by a retroviral vector. First, the vector must reach the cell surface where the receptors reside. Second, the virion must bind to its cognate receptor. 
Third, the viral envelope must fuse with the host cell envelope. Following these steps, the nucleocapsid enters the cell cytoplasm where additional steps, including reverse transcription and translocation of the pre-integration complex to the nucleus occur. In previous studies, we documented that MuLV-based vectors pseudotyped with the amphotropic envelope infect human, ${ }^{9}$ rabbit, ${ }^{17}$ and rat $^{10}$ airway epithelia from the basolateral, but not the apical surface. In the present study, we investigated the localization of the Pit2 receptor in polarized human airway epithelia using immunohistochemistry and the expression of a GFP-tagged Pit2 protein. Binding of amphotropic MuLV to the apical surface of airway epithelia was investigated using radiolabeled virus. Furthermore, we characterized components of the apical surface glycocalyx in human airway epithelia and investigated whether modifications of the abundance and composition of this potential physical barrier might enhance gene transfer efficiency.

\section{Results}

\section{The amphotropic receptor (Pit2) is expressed on the apical and basolateral membranes of differentiated human airway epithelia}

Our previous studies showed that MuLV pseudotyped with the amphotropic envelope was inefficient at transducing proliferating epithelia when applied to the apical surface. ${ }^{9,10}$ We hypothesized that absence of amphotropic Pit2 receptor on the apical membrane might account for this inefficiency. To test the hypothesis, we performed immunostaining to localize the human Pit2 receptor. The Pit2 polyclonal antibody was applied to either the apical or basolateral surface of the epithelia. As shown by confocal microscopy in Figure $1 \mathrm{a}, \mathrm{b}, \mathrm{f}, \mathrm{g}$, the Pit 2 receptor is expressed on both the apical and the basolateral surfaces. In addition, some intracellular signal was also detected.

To confirm this unexpected result, we made a fusion construct of human Pit2 and eGFP, and expressed this protein in epithelia using a first generation adenoviral vector (Ad5-CMVPit2-eGFP). Differentiated airway epithelial cells were transduced from the basolateral surface with this vector (50 MOI). Two days later, confocal microscopy demonstrated that the eGFP-tagged Pit2 receptor was distributed on both the apical and basolateral membrane domains (Figures $1 c-e, h-j$ ). In addition to the GFP signal on the apical and basolateral membranes, a diffuse intracellular signal was detected. These studies demonstrate that Pit2 is expressed on both the apical and basolateral membranes of human airway epithelia.

One possible explanation for the failure of the amphotropic enveloped MuLV to transduce airway epithelia despite the presence of Pit2 on the apical membrane is that Pit2 abundance might not be sufficient to support gene transfer. To address this possibility, we transduced airway epithelia with Ad5-CMVPit2-eGFP and then applied the amphotropic MuLV vector to the apical surface. To demonstrate that the Pit2-eGFP construct is functional, amphotropic vector resistant $\mathrm{CHO}$ cells were transduced with 50 MOI of Ad5-CMVPit2-eGFP. As shown in Figure 2a, CHO cells transduced with the Pit2expressing adenoviral vector were susceptible to gene transfer with the TA7- $\beta$ gal amphotropic pseudotyped vector, while the parental cells were not. This confirms that the Pit2-eGFP protein is functional as a virus receptor and the expression level following adenovirus infection is sufficient to support amphotropic retroviral transduction. However, when we transduced airway epithelia with Ad5-CMVPit2-eGFP and 3 days later applied TA7Bgal vector to the apical surface of KGF-treated cells, airway cells remained resistant to transduction (Figure $2 b-$ d). From these experiments, we conclude that the apical resistance to amphotropic MuLV transduction is not due to an absence of Pit2 expression.

\section{Apically applied amphotropic MuLV binds to Pit2 on the apical surface of airway epithelia}

Amphotropic MuLV vector binds specifically to the apical Pit2 receptor, an inorganic phosphate transporter. ${ }^{18}$ We used radiolabeled MuLV to ask whether the virus binds to the apical surface of airway epithelia in a fashion consistent with Pit2 binding. 20 MOI of the ${ }^{33} \mathrm{P}$-uridinelabeled vector was applied to the apical surface and incubated for $45 \mathrm{~min}$ at $4^{\circ} \mathrm{C}$. Following four washes with cold saline, the membrane with the attached epithelia was removed and placed in $1 \%$ Triton X-100. Then, the samples were transferred to scintillation cocktail and the radioactivity counted. As shown in the left-most bar in Figure 3, the radiolabeled ampho-MuLV vector binds to the apical surface. Importantly, this binding is blocked by goat anti-gp70 antiserum (second bar), but not normal goat serum (first bar). Since Pit2 is a sodium-dependent phosphate transporter, we used inorganic phosphate as a competitive inhibitor of Pit2 binding as described by others. ${ }^{19}$ When $40 \mathrm{mM} \mathrm{NaH}{ }_{2} \mathrm{PO}_{4}$ was included in the solution with the apically applied $\mathrm{MuLV}$, binding was inhibited (third bar). In contrast, $40 \mathrm{mM} \mathrm{NaHCO}_{4}$ had no such inhibitory effect on binding (fourth bar). These data suggest that apically applied amphotropic MuLV binds to Pit2.

\section{Characterization of physical barriers on the apical surface of well-differentiated human airway epithelia}

To begin to characterize components on the apical surface of human airway epithelia that might act as physical barriers to gene transfer, we first examined the surface by scanning and transmission electron microscopy. As demonstrated by SEM in Figure $4 \mathrm{a}$, a dense network of material was present around and in between the microvilli and cilia. In some areas between microvilli, spherical bodies were seen suggestive of glycocalyceal bodies. ${ }^{20}$ Ultrathin TEM sections revealed a fuzzy, electron dense material distributed between cilia and microvilli on the apical surface (Figure $4 \mathrm{~b}$ ). The electron dense material is in close apposition to the microvilli and extends out into the space between microvilli. This morphology is consistent with a glycocalyx on the apical surface of human airway cells. In addition, secreted products of the cells might also contribute to this meshwork on the apical surface.

The apical airway surface glycocalyx is characterized by the presence of many glycoproteins. ${ }^{20-23}$ To identify some of the components of the apical glycocalyx on human epithelia, we performed immunohistochemistry and ligand binding studies, focusing on three candidates: sialic acid, keratan sulfate and collagen type V. Sialic acids are terminal components of many glycoproteins and glycolipids. ${ }^{24}$ Wheat germ agglutinin (WGA) was 

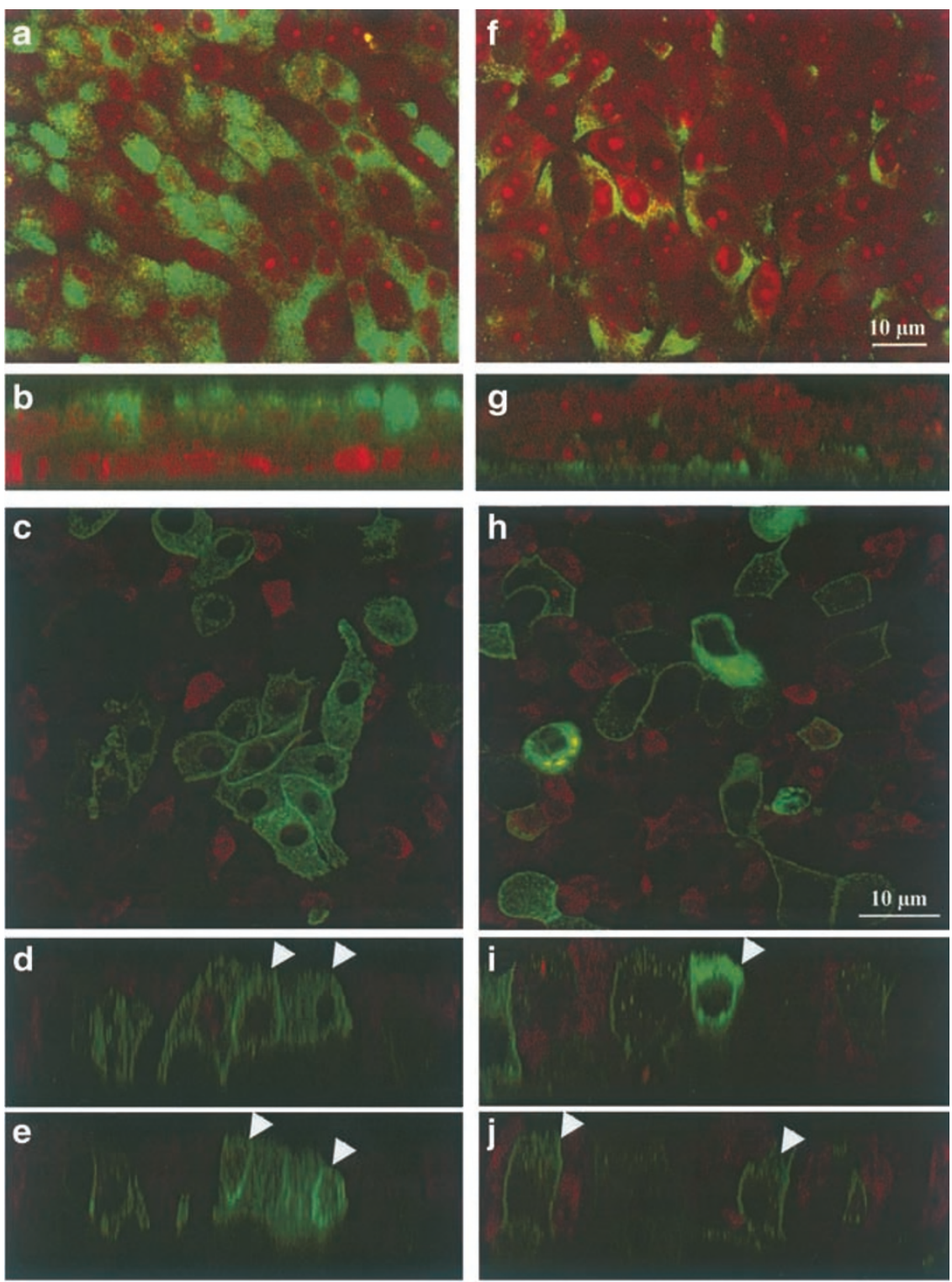

Figure 1 Localization of Pit2 receptor expression on the apical and basolateral membranes of differentiated human airway epithelia. $(a, b, f, g)$ Confocal micrographs of Pit2 immunolocalization on the apical and basolateral surfaces of polarized epithelia. $(a, b)$ Apical application of Pit2 antibody. (f, g) Basolateral application of Pit2 antisera. ( $a$ and $f) X-Y$ sections. ( $b$ and $g$ ) X-Z sections. Pit2 expression (FITC signal, green) is detected on both the apical and basolateral surfaces. Propidium iodide (red signal) counterstains nuclei and cytoplasm. (c-e and h-j) Confocal micrographs of airway epithelia expressing a Pit2-eGFP fusion receptor protein. Airway epithelia were infected with 50 MOI of Ad5CMVPit2-eGFP vector from the basolateral surface. Forty-eight hours later cells were examined for GFP localization by confocal microscopy $(c-e$, view $1 ; h-j$, view $2 ; c$ and $h, X-Y$ sections; $d, e, i$ and $j$, $X-Z$ sections). Pit2-eGFP expression (green signal) is seen on both the apical and basolateral membranes, as well as in the cytoplasm. Arrowheads indicate apical Pit2 signal. Views shown are representative of two different epithelial cell preparations examined.

used as a probe to detect all sialic acid linkages. Keratan sulfate is a common component of sulfated glycosaminoglycans, which covalently binds to core proteins, while collagen type $\mathrm{V}$ is a typical core protein found in the basement membrane of the airway epithelium and is secreted. ${ }^{25-27}$ Antibodies against keratan sulfate and collagen $\mathrm{V}$ were applied to the apical surface of airway epithelia to detect their respective antigens.

FITC-WGA was applied to the apical surface and the airway epithelia were examined by confocal microscopy. As shown in Figure 5a and b, the apical surface exhibited a diffuse WGA signal, consistent with the apical expression of sialic acid moieties. There was heterogeneity in the expression pattern, with some cells staining densely, and others more lightly. The keratan sulfate antibody decorated the apical surface of some, but not all airway epithelial cells with variable intensity (Figure $5 c$ and $\mathrm{d}$ ). In contrast, the collagen $\mathrm{V}$ antibody gave a more diffuse, even staining pattern (Figure 5e and f). When examined by immunogold transmission electron microscopy, a fibrous network of structures on the apical surface was labeled with the anti-collagen $\mathrm{V}$ antibody (data not shown). This immunolabeled structure was identical to the fuzzy material demonstrated by conven- 
a
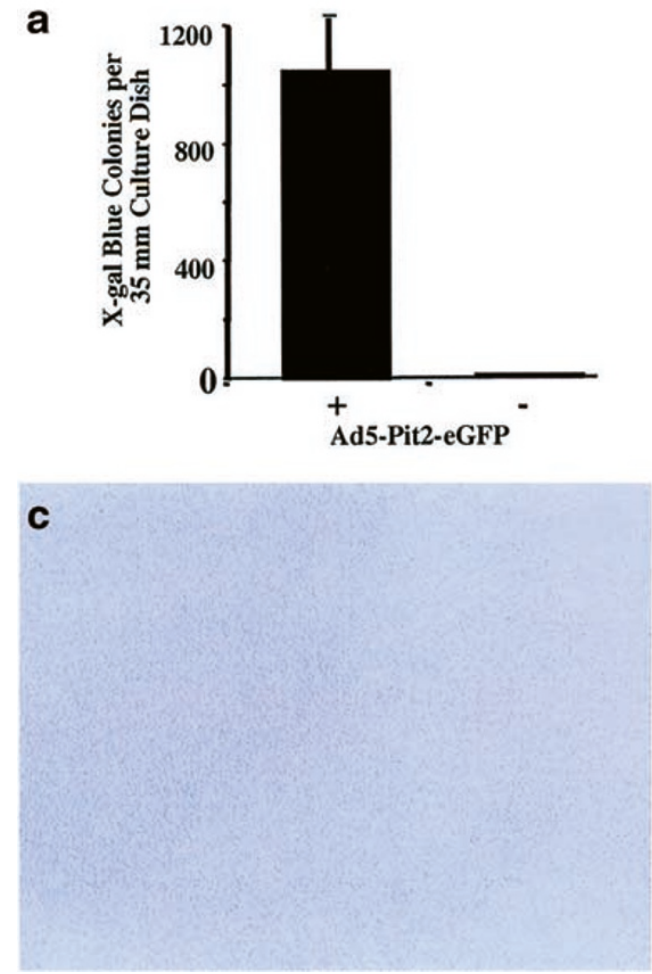
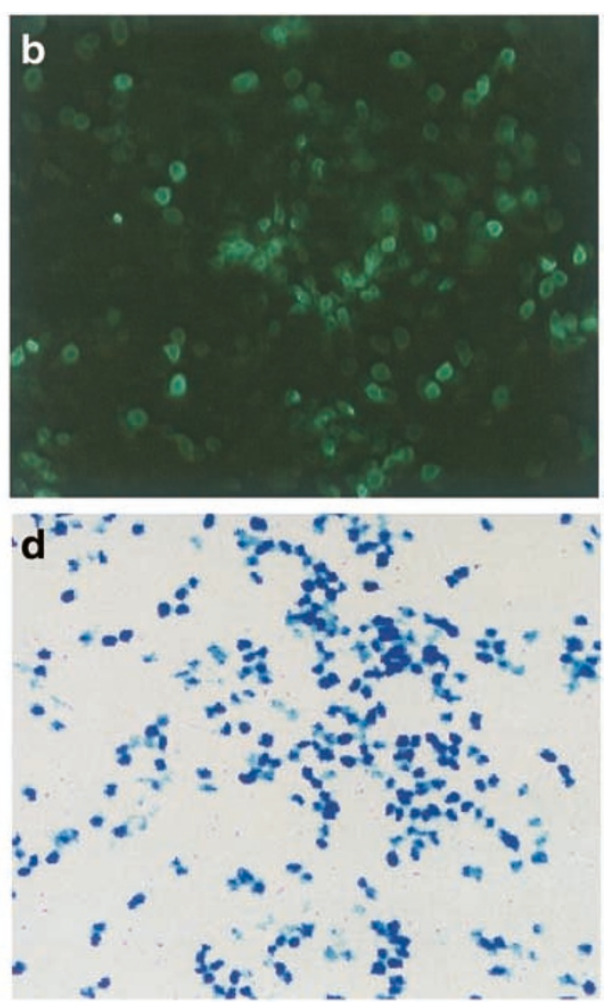

Figure 2 Ad5-CMVPit2-eGFP vector transduction confers expression of functional GPF tagged Pit2. CHO cells were pre-infected with 50 MOI of Ad5-CMVPit2-eGFP $24 \mathrm{~h}$ before ampho MuLV infection. In negative controls, Ad5-CMVPit2-eGFP was omitted. Three days after the ampho MuLV infection, X-gal staining was performed to evaluate the transduction efficiency. As shown in panel (a), the pre-infection with Ad5-CMVPit2-eGFP reversed the insusceptibility of $\mathrm{CHO}$ cells to ampho-MuLV, demonstrating that the Pit2-eGFP fusion construct is functional. Similarly, we infected KGF-stimulated human airway cells with 50 MOI of Ad5-CMVPit2-eGFP. As seen in panel (b), a large population of airway cells expresses the Pit2eGFP protein. However, when $20 \mathrm{MOI}$ of ampho MuLV was applied to the apical surface, no gene transfer was noted after X-gal staining (c). If the $M u L V$ vector was formulated with EGTA to disrupt tight junctions, efficient gene transfer was achieved (d).

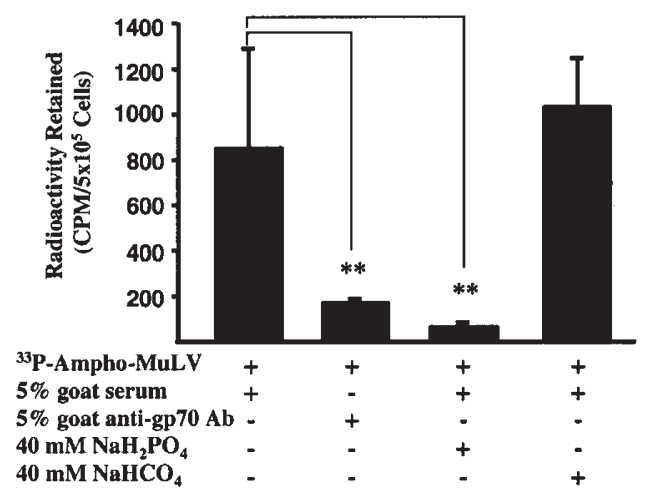

Figure 3 Amphotropic $M u L V$ vector binds specifically to the apical Pit2 receptor. The amphotropic $M u L V$ vector was labeled with ${ }^{33} P$-uridine as described in Materials and methods and applied to the apical surface under several conditions. As shown in the left-most bar in the figure, the radiolabeled vector binds to the apical surface. This binding was blocked by goat anti-gp70 antiserum (second bar), but not normal goat serum (first bar). Furthermore, $40 \mathrm{mM} \mathrm{NaH}{ }_{2} \mathrm{PO}_{4}$ inhibited binding of amphotropic $\mathrm{MuLV}$ binding (third bar). In contrast, $40 \mathrm{mM} \mathrm{NaHCO}$ had no inhibitory effect (fourth bar). **, Indicates statistically significant difference compared with amphotropic $M u L V+$ normal goat serum condition $(P<0.05, t$ test). Representative figure of four similar experiments. tional TEM in Figure 4b. These data indicate that sialic acids, keratan sulfate and collagen type V are components of the apical glycocalyx.

\section{Modification of apical glycocalyx components}

We hypothesized that the glycocalyx might present a physical barrier and prevent amphotropic pseudotyped retrovirus from binding to Pit2 on the apical surface. If the dense matrix could be removed or modified, perhaps the vector could reach the receptor. Various approaches to modifying the apical surface with enzymes have been used previously in attempts to increase adenoviral and AAV gene transfer efficiency. ${ }^{4,7}$ In a pilot experiment, we found that treatment of the apical surface with neuraminidase followed by application of TA7-Bgal vector did not enhance gene transfer.

To modify the glycocalyx, we treated the apical surface of differentiated airway epithelia with heparinase I and III, chondroitinase ABC, and collagenase (see Materials and methods). Having previously documented the presence of sialic acids, keratan sulfate and collagen type $\mathrm{V}$ on the surface, the presence of these components was used to judge the efficacy of the enzyme treatment. As shown in Figure $5 \mathrm{~g}$ to 1 , the enzyme treatment diminished the apical keratan sulfate and collagen V components, but did not alter sialic acid abundance. Since the sialic acids are linked to membrane glycoproteins and glycolipids, we speculate that the enzymatic removal of some glycocalyx components exposed the membrane- 

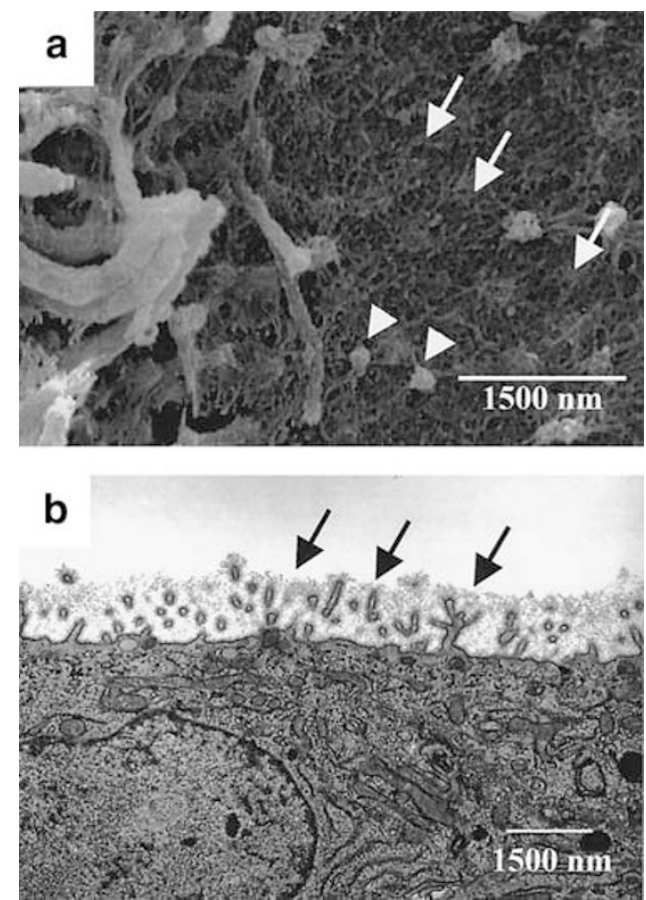

Figure 4 Morphology of the apical surface of well-differentiated human airway epithelia. (a) SEM view of the apical surface. A dense fibrous network was noted around cilia and microvilli on the apical surface (arrows). Spherical bodies suggestive of glycocalyceal bodies were also seen (arrow heads). (b) Ultra-thin sections of the airway epithelia were examined by transmission electron microscopy. The fuzzy apical glycocalyx around and in between the microvilli is indicated by the arrows. Views shown are representative of three different epithelial cell preparations examined.

linked sialic acid binding sites. Therefore, the sialic acid staining signal as measured by WGA lectin binding was not decreased. To confirm that the enzymes removed portions of the apical glycocalyx, we also examined the apical surface by scanning and transmission electron microscopy after the treatment. As shown in Figure $5 \mathrm{~m}$ and $n$, these electron micrographs demonstrate that the abundance of the apical glycocalyx meshwork was greatly reduced by the enzymes (contrast with untreated cells shown in Figure 1).

\section{Enzyme treatment of the apical surface decreases the transepithelial resistance}

Following enzyme treatment, epithelial integrity was evaluated by measuring the transepithelial resistance. We observed that enzyme-exposed cells promptly had a significant drop in transepithelial resistance to $\sim 35 \%$ of control values that slowly recovered over many hours (Figure 6). Such disruption of epithelial integrity might allow apically applied viral vectors to reach the basolateral surface through the opened epithelial junctions. ${ }^{5,9,17,28}$ For this reason, it is difficult to evaluate the effect of direct enzymatic modifications of the apical glycocalyx on gene transfer efficiency by performing gene transfer immediately following enzyme treatment.

\section{Enzyme treatment followed by inhibition of glycosylation maintains apical surface glycocalyx modifications and allows transepithelial resistance to recover}

In addition to simple enzymatic modification of the apical surface, we evaluated whether culturing the cells in the presence of 2-deoxy-D-glucose altered apical surface morphology. 2-deoxy-D-glucose, an inhibitor of glycosylation, has been reported to inhibit the formation of lipidlinked oligosaccharides. ${ }^{29}$ The glycocalyx is known to be highly glycosylated. Therefore, we used this reagent to inhibit the normal production of new proteoglycans and glycosaminoglycans necessary for the formation of the glycocalyx. We hypothesized that inhibiting glycosylation after enzyme digestion might block the formation of a new glycocalyx meshwork on the apical surface. To test this hypothesis, we first treated the apical surface of differentiated airway epithelia with the enzymes for $2 \mathrm{~h}$ at $37^{\circ} \mathrm{C}$. Subsequently, $100 \mathrm{mM}$ 2-deoxy-D-glucose was included in the cell culture medium. Following enzyme treatment and culture with 2-deoxy-D-glucose, the transepithelial resistance was measured and the apical surface was examined by scanning electron microscopy. As shown in Figure 7a, the transepithelial resistance recovered to control levels 5 days after culture in the 2-deoxyD-glucose-containing culture medium. Scanning and transmission electron microscopy on day 5 demonstrated that the apical glycocalyx remained reduced in abundance (Figure $7 b, c)$. Immunostaining for keratan sulfate and collagen $\mathrm{V}$ showed that most of the surface area stained weakly, compared with the untreated controls, while sialic acid abundance was unchanged, essentially identical to the results shown in Figure 5g-1 (data not shown). Thus, this approach modifies the apical surface, while allowing epithelial integrity to recover.

\section{Modification of the glycocalyx fails to enhance gene transfer to airway epithelia}

To determine whether the apical glycocalyx constitutes a barrier impeding apical gene transfer with amphotropic MuLV, we applied $20 \mathrm{MOI}$ of amphotropic MuLV $\beta$-gal to the apical surface of airway epithelia with or without modification of the glycocalyx. Before vector transduction, the airway cells were stimulated with KGF for $24 \mathrm{~h}$, as described previously., 10 Three days after gene transfer, the transduction efficiency was examined by X-gal histochemical staining. As shown in Figure 8, in control epithelia (Figure 8a) or epithelia treated with enzymes and then maintained in 2-deoxy-D-glucose-containing media for 5 days (Figure 8b), amphotropic MuLV failed to transduce cells following apical application. As a positive control, enzyme and 2-deoxy-D-glucose-treated airway epithelia were transduced apically with vector formulated with EGTA. We previously showed that EGTA opens epithelial junctions, allowing the apically applied MuLV vectors access to the susceptible basolateral surface. ${ }^{9,10,17}$ Figure $8 \mathrm{c}$ shows that the EGTA formulated amphotropic MuLV vector efficiently transduces the epithelia, confirming that the Pit2 receptor is functional. Thus, despite significant modifications of the glycocalyx, gene transfer from the apical surface was not enhanced.

To rule out the possibility that the enzyme treatment and 2-deoxy-D-glucose culture conditions altered the distribution of Pit2, the apical surface of airway epithelia was immunostained for Pit2 immediately following enzyme treatment, or after culture in 2-deoxy-D-glucosecontaining media for 5 days after enzyme treatment. Confocal microscopic examination demonstrated that the enzyme treatment alone, or the combined enzyme treatment and 2-deoxy-D-glucose culture conditions did not 

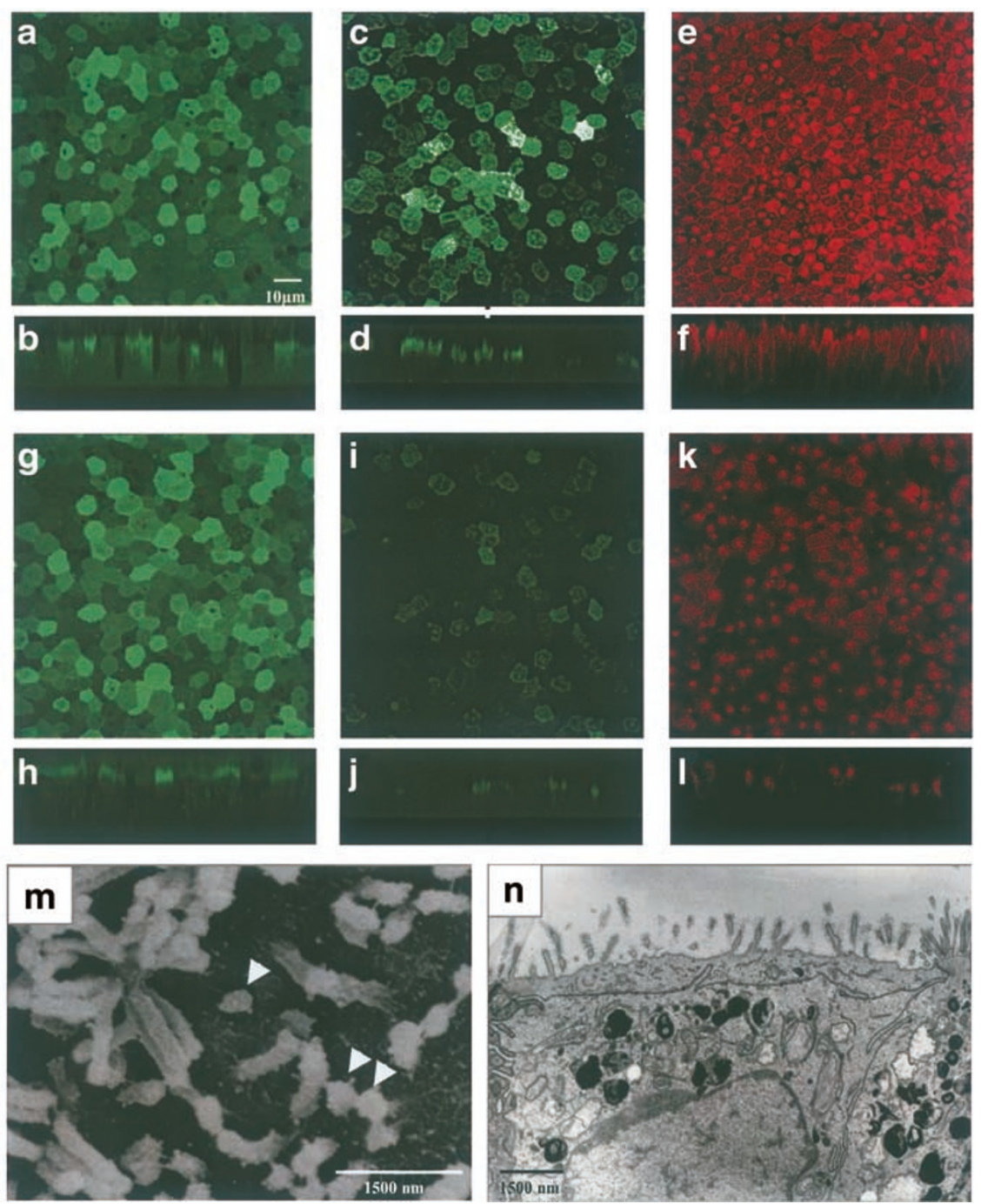

Figure 5 Components of the apical glycocalyx and surface alterations produced by enzyme treatment. Confocal micrographs (a-l). Apical surface staining for sialic acids in control airway cells $(a, b)$ and enzyme-treated airway cells $(g, h)$ with WGA-FITC as described in Materials and methods. Apical keratan sulfate staining of the control airway epithelia $(c, d)$ and enzyme-treated cells $(i, j)$. Apical localization of collagen type $V$ in control $(e, f)$ and enzyme-treated airway epithelia $(k, l)$. X-Y sections $(a, c, e, g, i$ and $k) ; X-Z$ sections $(b, d, f, h, j$ and $l) ;(m-n)$ Airway epithelia treated with enzymes were examined by scanning and transmission electron microscopy; $m$, scanning EM image shows reduction in the fibrous network of the apical glycocalyx. Some spherical bodies suggestive of glycocalyceal bodies remain (arrow heads). $n$, Transmission EM image demonstrates that the fuzzy material around microvilli and cilia was much reduced. Contrast panels $(m)$ and $(n)$ with images in Figure 4. Views shown are representative of three different epithelial cell preparations examined.

alter the distribution of Pit2 on the apical surface (not shown).

\section{Discussion}

The present study investigated some of the barriers to gene transfer with the amphotropic envelope pseudotype when the retroviral vector is applied to the apical surface of well-differentiated human airway epithelia. In contrast to the findings for the coxackie $\mathrm{B}$ and adenovirus serotype 2 and 5 receptor (CAR), where the receptor expression is polarized to the basolateral surface, $, 4,5$ we found that Pit2 is present on both the apical and basolateral surfaces of airway epithelia. Furthermore, these studies indicate that the amphotropic MuLV vector binds to the apical surface of airway epithelia and that this binding is reduced in the presence of $\mathrm{NaH}_{2} \mathrm{PO}_{4}$, suggesting it is mediated by Pit2. ${ }^{19}$ Therefore the limitation for apical gene transfer with amphotropic retrovirus is more than the simple absence of the appropriate cellular receptor. Increasing Pit2 expression in airway epithelial cells with an adenoviral vector did not enhance apical gene transfer with the amphotropic retrovirus either, suggesting that the abundance of Pit2 is not the limitation. Potential physical barriers that might prevent the vector from reaching apical receptors are the glycocalyx and other secreted, adherent products of epithelia. Our studies indicate that the apical surface of human airway epithelia has a dense meshwork of material that is morphologically compatible with a glycocalyx. Furthermore, some of the components of this matrix were identified histochemically to include sialic acid, keratan sulfate, and collagen type V. Attempts to enhance gene transfer by removing apical glycocalyx components with enzymes or preventing its formation by growing cells in 2-deoxy-D-glucose-containing media were unsuccessful. The results 


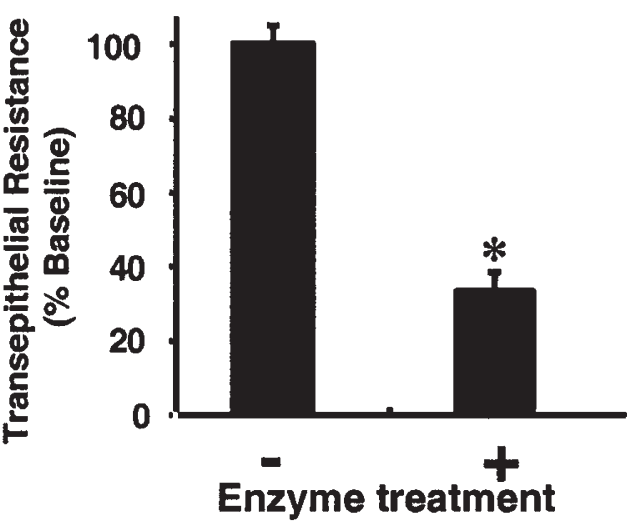

Figure 6 Enzyme treatment significantly decreases the transepithelial resistance. Following enzyme treatment for $2 \mathrm{~h}$ at $37^{\circ} \mathrm{C}$, the transepithelial resistance was measured as described in Materials and methods. As compared with controls, this treatment reduced the transepithelial resistance to $\sim 35 \%$ of the controls. The transepithelial resistance of enzyme-treated cells gradually recovered to baseline values after 4-5 days (data not shown). Results shown represent mean \pm s.e. $*$, Indicates statistically significant difference compared with controls $(P<0.05$, $t$ test, $n=4)$.

suggest that factors other than Pit2 receptor abundance limit gene transfer from the apical surface of airway epithelia.

The glycocalyx is an intricate network of locally produced glycoproteins, glycolipids and proteoglycans that are assembled into an organized structure and attached to the cell surface. ${ }^{20-23}$ This meshwork constitutes the outermost surface of a cell that a retroviral vector must cross in order to gain access to receptors for binding and fusion. While the glycoproteins comprising a glycocalyx are typically heavily modified by sialation and sulfation, the composition of the glycocalyx on the apical surface of human airway epithelia is not well characterized. Our studies confirm that glycoproteins modified by the addition of sialic acid moieties and keratan sulfate are present on the apical surface. In addition to this attached glycocalyx, epithelia may secrete other products that also act as physical barriers to gene transfer. These products include mucins ${ }^{30}$ and extracellular matrix components, such as collagen and fibronectin. ${ }^{31}$ It was previously shown that the basement membrane of the airway epithelium contains several extracellular matrix molecules including chondroitin sulfate, heparan sulfate proteoglycan, entactin, laminin, fibronectin, and type $\mathrm{V}$ collagen. ${ }^{25,32}$ The present studies indicate that the apical surface of airway epithelia also contains type $\mathrm{V}$ collagen. This type of collagen self-assembles and may contribute to the barrier properties of the apical surface.

Enzymatic modifications of the apical surface have previously been investigated as a means to enhance gene transfer with non-retroviral vector systems. Pilewski and colleagues found that human airway epithelia or MDCK cells expressing the mucin gene MUC1, a complex sialated glycoconjugate attached to the cell surface, were resistant to gene transfer with adenoviral vectors. ${ }^{33}$ This inhibition was overcome by treating cell surfaces with neuraminidase to remove sialic acid moieties. In studies of AAV2-mediated gene transfer to airway epithelia, Bals and colleagues ${ }^{7}$ reported that treatment of the apical surface of differentiated airway epithelial cells with various enzymes including trypsin, $\mathrm{N}$-glycosidase $\mathrm{F}$, neuraminid-
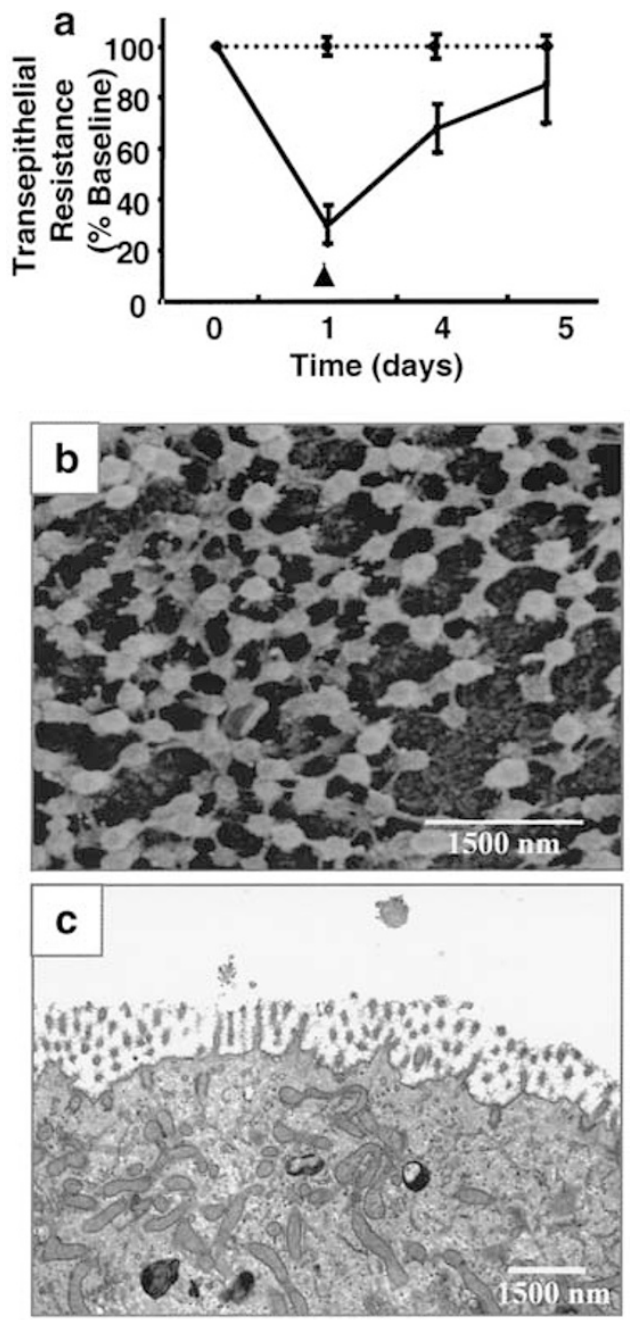

Figure 7 Glycosylation inhibition following enzyme treatment allows transepithelial resistance, but not the apical glycocalyx, to recover. 100 mM 2-deoxy-D-glucose was applied to the culture medium after the apical surface of differentiated airway epithelia was digested enzymatically. Transepithelial resistance was measured each day for 5 days. (a) In enzyme-treated cells transepithelial resistance gradually recovered to control levels 5 days after treatment. Results shown represent mean \pm s.e. (b) Scanning electron microscopy demonstrates that the apical surface remained modified after enzyme treatment and culture in 2-deoxy-D-glucose-containing media. The fibrous network of the apical glycocalyx remains reduced. Some spherical bodies suggestive of glycocalyceal bodies remain. (c) Transmission EM similarly shows that the glycocalyx is largely under these conditions. Views shown are representative of three different epithelial cell preparations examined.

ase, N-glycosidase $\mathrm{A}$, and endoglycosidase $\mathrm{H}$ enhanced vector transduction from the apical surface. In the same study, the addition of mucin or heparin inhibited gene transfer. Pickles and coworkers ${ }^{4}$ investigated the effects of enzymatic apical surface modifications on the apical gene transfer efficiency of serotype 5 adenovirus vectors. Polarized MDCK cells expressing GPI-linked CAR were used as a model. Enzymatic modification with neuraminidase, trypsin, keratanase, or elastase enhanced apical gene transfer to MDCK cells expressing apical CAR. ${ }^{4}$ In contrast to these findings, Walters et $\mathrm{ll}^{34}$ recently reported that expression of GPI-linked CAR on the apical surface of polarized human airway epithelia was sufficient to confer apical infectivity with serotype 5 adenovirus. 


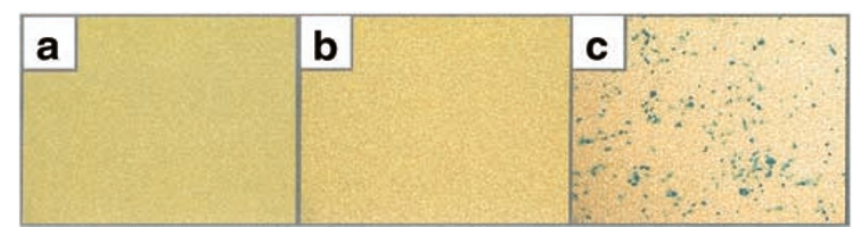

Figure 8 Modification of the apical glycocalyx fails to facilitate gene transfer from the apical surface with amphotropic MuLV vector. All cells were pretreated with KGF to stimulate proliferation. Views shown are low, power en face photomicrographs of X-gal stained epithelia. (a) 20 MOI of amphotropic MuLV was applied apically to control airway epithelia and no significant gene transfer occurred. (b) Enzyme and 2-deoxyD-glucose-treated airway epithelia were transduced apically with 20 MOI of the amphotropic retroviral vector. There was no evidence of gene transfer. (c) Enzyme and 2-deoxy-D-glucose-treated airway epithelia were transduced apically with $20 \mathrm{MOI}$ of the amphotropic MuLV vector formulated with $6 \mathrm{mM}$ EGTA. X-gal positive (blue) cells representing gene transfer were only noted in cells treated with EGTA formulated vector. The experiment was performed three times with two different epithelial cell preparations.

Further removal of glycocalyx components with neuraminidase or glyosidases did not enhance adenoviral gene transfer. ${ }^{34}$

In contrast to these results with AAV2 and adenoviral gene transfer, the present studies indicate that the Pit2 receptor is expressed on both membrane domains of human airway epithelia. Despite the expression of Pit2 on the apical surface of airway epithelia, it does not support gene transfer with amphotropic retrovirus, with or without enzymatic modifications of the surface. Therefore, we conclude that factors other than receptor abundance limit amphotropic retrovirus gene transfer. There are several possible explanations for these results that require further study. The Pit 2 protein that is sorted to the apical membrane may not functionally support viral entry while basolaterally sorted Pit 2 does. It is also possible that there are additional co-receptors or co-factors required for infection via Pit 2 and that these are missing from the apical surface. Furthermore, we have not yet determined whether fusion does occur for the apically applied vector. If fusion does occur, it is possible that steps that occur after the nucleocapsid enters the cytoplasm, such as reverse transcription and trafficking to the nucleus, are not equivalent following entry from the apical and basolateral cell surfaces. ${ }^{35}$

It is important to note that that these findings with the amphotropic pseudotype can not be generalized to all enveloped viruses. Using the same model of primary human airway epithelia, we recently demonstrated that wild-type human coronavirus $229 \mathrm{E}^{36}$ and influenza virus (A/Japan/305/57 (H2N2) $)^{37}$ both infect polarized airway epithelia when applied to the apical surface. Similar results were seen with wild-type respiratory syncytial virus and parainfluenza virus type 3 (McCray and Sinn, unpublished observations). Clearly some respiratory viruses evolved methods to efficiently bypass apical barriers on airway epithelia. These properties of apical infection might be adopted to improve current retroviral vector systems for airway gene transfer. Thus we conclude that there is no evidence of an absolute barrier to apical airway gene transfer for enveloped viruses.

We conclude that the glycocalyx is unlikely to be a major impediment to apical gene transfer with amphotropic MuLV. This finding focuses the search for apical barriers to amphotropic retroviral gene transfer to sub-

sequent events, such as fusion, or steps beyond entry. Future efforts to delineate apical barriers to retroviral gene transfer will focus on these later steps of the viral entry process.

\section{Materials and methods}

\section{Cell culture}

Human airway epithelial cells were obtained from the tracheas and bronchi of lungs removed from donated organs. Cells were isolated by enzymatic dissociation as previously described. ${ }^{38,39}$ Freshly isolated cells were seeded at a density of $5 \times 10^{5}$ cells $/ \mathrm{cm}^{2}$ on to collagencoated $0.6 \mathrm{~cm}^{2}$ diameter Millicell polycarbonate filters (Millipore, Bedford, MA, USA). The cells were maintained at $37^{\circ} \mathrm{C}$ in a humidified atmosphere of $7 \% \mathrm{CO}_{2}$ and air. Twenty-four hours after plating, the mucosal medium was removed and the cells were allowed to grow at an air-liquid interface. ${ }^{38}$ The culture medium consisted of a 1:1 mix of DMEM/Ham's F12, 5\% Ultroser $\mathrm{G}$ (Biosepra, France), $100 \mathrm{U} / \mathrm{ml}$ penicillin, $100 \mu \mathrm{g} / \mathrm{ml}$ streptomycin, $1 \%$ nonessential amino acids, and 0.12 $\mathrm{U} / \mathrm{ml}$ insulin. Studies were performed on well-differentiated cells ( $>2$ weeks old). This study was approved by the Institutional Review Board at the University of Iowa.

\section{Localization of the Pit2 receptor in airway epithelia}

To determine the localization of the amphotropic receptor Pit2, two approaches were taken. To immunolocalize Pit2, airway epithelia were fixed in $4 \%$ paraformaldehyde for $20 \mathrm{~min}$ at room temperature. After two, 10-min PBS washes, the cells were blocked in 5\% BSA overnight at $4^{\circ} \mathrm{C}$. A polyclonal rabbit antibody to human Pit 2 was kindly provided by Dr Helmuth HG van ES (IntroGene, Leiden, The Netherlands). ${ }^{9}$ The primary antibody to human Pit2 was diluted 100-fold. The primary antibody was added to either the apical or basolateral cell surface and subsequently applied an FITC conjugated goat antirabbit secondary antibody (Sigma, St Louis, MO, USA, 500-fold dilution). A propidium iodide stain was then applied to counterstain the nuclei and cytoplasm. Following immunostaining, the samples were examined with either epifluorescent (BX60, Olympus) or confocal microscopy (MRC 1024, BioRad, Hercules, CA, USA).

As a second approach to localize Pit2 expression in airway epithelia, we constructed a chimeric protein consisting of human Pit2 fused to eGFP. The human Pit2 cDNA was a generous gift of Jean M Heard. ${ }^{19}$ Enhanced GFP (Clontech, Palo Alto, CA, USA) was placed at the N terminus of the human Pit2 cDNA. This construct was then cloned into an adenovirus shuttle plasmid in which the expression of eGFP/human Pit2 fusion was driven by the CMV promoter. The construct was packaged into an E1-deleted Ad5 vector as previously described ${ }^{40}$ and a replication defective virus produced in the University of Iowa Gene Transfer Vector Core. Fifty MOI of the resultant Ad5-CMVPit2-eGFP vector was used to transduce airway epithelia and investigate the distribution of Pit2.

\section{Pit2 binding studies using radiolabeled amphotropic- \\ MuLV}

Amphotropic retrovirus was radiolabeled with ${ }^{33} \mathrm{P}$-uridine (Amersham) by adding the compound to the cell 
culture media of the TA-7ßgal-packaging cells (described below). TA-7 $\beta$ gal cells were plated at a density of $10 \times$ $10^{6}$ cells per $150-\mathrm{mm}$ tissue culture plate. Twenty-four $\mathrm{h}$ later, the cell culture media (DMEM, 10\% fetal bovine serum, $100 \mathrm{U} / \mathrm{ml}$ penicillin, $100 \mathrm{mg} / \mathrm{ml}$ streptomycin) was replaced with fresh medium containing $10 \mathrm{mCi} / \mathrm{ml}$ of ${ }^{33} \mathrm{P}$-uridine. The virus was harvested from the cell culture supernatants and filtered and concentrated by centrifugation by routine procedures. The resultant labeled preparation maintained an infectious titer similar to the unlabeled preparations. To study binding of the labeled amphotropic-MuLV vector to airway epithelia, $20 \mathrm{MOI}$ of the labeled vector was applied to the apical surface and incubated for $45 \mathrm{~min}$ at $4^{\circ} \mathrm{C}$. Following four washes with cold saline, the epithelia were lysed in $1 \%$ Triton X-100 and binding assayed by scintillation counting. To demonstrate specificity of the binding to Pit2, the experiment was performed in the presence or absence of goat antigp70 antisera, ${ }^{41}$ normal goat sera, $40 \mathrm{mM} \mathrm{NaH}{ }_{2} \mathrm{PO}_{4}{ }^{19}$ or $40 \mathrm{mM} \mathrm{NaHCO}$. Each experimental condition was performed in quadruplicate.

\section{Characterization of the apical glycocalyx}

Transmission and scanning electron microscopy: To characterize the morphology of the apical surface of airway epithelia, the cells were examined by scanning and transmission electron microscopy. Epithelia were fixed for transmission electron microscopy overnight at $4^{\circ} \mathrm{C}$ using $2.5 \%$ glutaraldehyde $(0.1 \mathrm{M}$ sodium cacodylate buffer, $\mathrm{pH} 7.2$ ), followed by post-fixation in $1 \%$ osmium tetroxide for $1 \mathrm{~h}$. Following serial dehydration in alcohols, samples were embedded in Eponate 12 (Ted Pella, Redding, CA, USA). Sectioning and post-staining were performed using standard methods. Samples were examined with a Hitachi H-7000 transmission electron microscope. Scanning EM samples were also fixed in $2.5 \%$ glutaraldehyde and post-fixed with $1 \%$ osmium tetroxide. Dehydration and critical point drying were performed following routine procedures. Samples were examined with a Hitachi S-4000 microscope.

Immunolocalization of glycocalyx components: Airway epithelia were first fixed in $4 \%$ paraformaldehyde for 20 min at room temperature. After two, 10-min PBS washes, the cells were blocked with $5 \%$ BSA overnight at $4^{\circ} \mathrm{C}$. Mouse anti-keratan sulfate antibody was purchased from Chemicon (MA2022, Temecula, CA, USA). Polyclonal rabbit antiserum against human collagen type $\mathrm{V}$ was purchased from CalBiochem (234190, La Jolla, CA, USA). The primary antibodies to keratan sulfate and human collagen V were diluted 500-fold and 100-fold, respectively. The secondary antibody (goat anti-rabbit or anti-mouse IgG conjugated to TRITC, Sigma) was diluted 300-fold. FITC-conjugated wheat germ agglutinin (WGA) was obtained from Sigma. Following immunostaining, the samples were mounted in media containing DAPI (Vector Laboratories, Burlingame, CA, USA) and examined with either epifluorescence (Olympus) or confocal microscopy (MRC 1024, BioRad).

Enzymatic treatment of the apical glycocalyx: To investigate the impact of physical modifications of the apical surface on the efficiency of retroviral gene transfer, we developed two methods. The first approach consisted of treating the apical surface with enzymes to remove glycocalyx components. Heparinase I (H-2519) and III (H8891), chondroitinase ABC (C-2905), and collagenase (C9407) were purchased from Sigma. The apical surface of differentiated airway cells was digested with the enzyme mixture $(50 \mathrm{U} / \mathrm{ml}$ heparinase $\mathrm{I}, 10 \mathrm{U} / \mathrm{ml}$ heparinase III, $10 \mathrm{U} / \mathrm{ml}$ chondroitinase ABC, and $1000 \mathrm{U} / \mathrm{ml}$ collagenase) for $2 \mathrm{~h}$ at $37^{\circ} \mathrm{C}$. As a measure of epithelial integrity, transepithelial resistance was measured before and after enzyme treatment using an ohmmeter (EVOM, World Precision Instruments, Sarasota, FL, USA) as described previously. ${ }^{9}$

The second method of apical surface modification consisted of growing the epithelia in the presence of 2-deoxyD-glucose to inhibit glycosylation of cell surface proteins. 2-deoxy-D-glucose (D-3179, Sigma) was added to the Ultroser G culture medium. Unless otherwise specified, following apical digestion with enzymes, airway epithelia were cultured in Ultroser $G$ medium containing 100 mM 2-deoxy-D-glucose for 5-7 days. Transmission and scanning electron microscopy were used to determine the efficacy of these treatments in modifying glyxcocalyx components and altering the apical surface morphology.

\section{Gene transfer}

A Moloney murine leukemia virus (MuLV)-based vector pseudotyped with the amphotropic envelope was used in these studies. The packaging cell line producing amphotropic enveloped, nuclear-targeted $\beta$-galactosidase MuLV retrovirus (TA-7ßgal) was kindly provided by Francois-Loic Cosset and was previously described. ${ }^{42,43}$ The reporter gene expression was driven by the MuLV LTR promoter. Titers obtained for the TA-7 $\beta$ gal vector were typically $1-5 \times 10^{8} \mathrm{TU} / \mathrm{ml}$ as determined by blue cell counts on HT1080 cells.

Before retroviral gene transfer, the differentiated airway cells were induced to proliferate by adding 100 $\mathrm{ng} / \mathrm{ml}$ of keratinocyte growth factor (KGF) to the culture media for 24 h. ${ }^{9,10}$ Twenty MOI of amphotropic MuLV was applied to the apical surface of airway cells as described previously. ${ }^{9,11}$ As a positive control, the vector was formulated with $6 \mathrm{mM}$ EGTA and applied apically to the enzyme and drug-treated airway cells. ${ }^{17}$ Three days after infection, the airway cells were stained for the $\beta$-galactosidase reporter gene expression using $X$-gal histochemistry.

\section{$X$-gal histochemistry}

Airway epithelia were fixed with $2 \%$ paraformaldehyde/ PBS solution for $20 \mathrm{~min}$ and rinsed with PBS twice for 5 min each. X-gal staining solution was added to the cells for $4 \mathrm{~h}$ at $37^{\circ} \mathrm{C}$ as previously described. ${ }^{9}$ Cells expressing $\beta$-galactosidase stained blue.

\section{Acknowledgements}

The authors gratefully acknowledge the technical expertise of Phil Karp and Pary Weber of the University of Iowa Cell Culture Core in preparing the human airway epithelial cultures. We thank Dr Yubin Kang for his helpful discussions. This work was supported in part by grants from the Cystic Fibrosis Foundation (GW99G0) and NIH (R01 HL61460 and P50 HL51670, BLD and PBM). We acknowledge the support of the Morphology Core and 
Gene Transfer Vector Core, partially supported by the Cystic Fibrosis Foundation, NHLBI (NIH P50 HL51670), and the Center for Gene Therapy for Cystic Fibrosis and other Genetic Diseases (NIH P30 DK54759).

\section{References}

1 Johnson LG et al. Efficiency of gene transfer for restoration of normal airway epithelial function in cystic fibrosis. Nature Genet 1992; 2: 21-25.

2 Wang G, Sinn PL, McCray Jr. PB. Development of retroviral vectors for gene transfer to airway epithelia. Curr Opin Mol Ther 2000; 2: 497-506.

3 Flotte TR et al. Stable in vivo expression of the cystic fibrosis transmembrane conductance regulator with an adeno-associated virus vector. Proc Natl Acad Sci USA 1993; 90: 10613-10617.

4 Pickles RJ et al. Retargeting the coxsackievirus and adenovirus receptor to the apical surface of polarized epithelial cells reveals the glycocalyx as a barrier to adenovirus-mediated gene transfer. J Virol 2000; 74: 6050-6057.

5 Walters RW et al. Basolateral localization of fiber receptors limits adenovirus infection of airway epithelia. J Biol Chem 1999; 274: 10219-10226.

6 Duan D, Yue Y, McCray Jr PB, Engelhardt JF. Polarity influences the efficiency of recombinant adeno-associated virus infection in differentiated airway epithelia. Hum Gene Ther 1998; 9: 2761-2776.

7 Bals $\mathrm{R}$ et al. Transduction of well-differentiated airway epithelium by recombinant adeno-associated virus is limited by vector entry. J Virol 1999; 73: 6085-6088.

8 Zabner J et al. Adeno-associated virus type 5 (AAV5) but not AAV2 binds to the apical surfaces of airway epithelia and facilitates gene transfer. J Virol 2000; 74: 3852-3858.

9 Wang G et al. Influence of cell polarity on retrovirus-mediated gene transfer to differentiated human airway epithelia. J Virol 1998; 72: 9818-9826.

10 Wang G et al. Keratinocyte growth factor induced epithelial proliferation facilitates retroviral-mediated gene transfer to pulmonary epithelia in vivo. J Gene Med 1999; 1: 22-30.

11 Wang $G$ et al. Feline immunodeficiency virus vectors persistently transduce nondividing airway epithelia and correct the cystic fibrosis defect. J Clin Invest 1999; 104: R49-R56.

12 Miller DG, Edwards RH, Miller AD. Cloning of the cellular receptor for amphotropic murine retroviruses reveals homology to that for gibbon ape leukemia virus. Proc Natl Acad Sci USA 1994; 91: 78-82.

13 Battini J, Rasko JEJ, Miller AD. A human cell-surface receptor for xenotropic and polytropic murine leukemia viruses: possible role in G protein-coupled signal transduction. Proc Natl Acad Sci USA 1999; 96: 1385-1390.

14 Tailor CS et al. Cloning and characterization of a cell surface receptor for xenotropic and polytropic murine leukemia viruses. Proc Natl Acad Sci USA 1999; 96: 927-932.

15 Yang YL et al. Receptors for polytropic and xenotropic mouse leukaemia viruses encoded by a single gene at Rmc1. Nat Genet 1999; 21: 216-219.

16 Schlegel R, Tralka TS, Willingham MC, Pastan I. Inhibition of VSV binding and infectivity by phosphatidylserine: is phosphatidylserine a VSV-binding site? Cell 1983; 32: 639-646.

17 Wang G et al. Increasing epithelial junction permeability enhances gene transfer to airway epithelia in vivo. Am J Respir Cell Mol Biol 2000; 22: 129-138.

18 Kavanaugh MP et al. Cell-surface receptors for gibbon ape leukemia virus and amphotropic murine retrovirus are inducible sodium-dependent phosphate symporters. Proc Natl Acad Sci USA 1994; 91: 7071-7075.

19 Rodrigues P, Heard JM. Modulation of phosphate uptake and amphotropic murine leukemia virus entry by posttranslational modifications of PIT-2. J Virol 1999; 73: 3789-3799.

20 Afzelius BA. Glycocalyx and glycocalyceal bodies in the respiratory epithelium of nose and bronchi. Ultrastruct Pathol 1984; 7: $1-8$.

21 Spicer SS, Mochizuki I, Setser ME, Martinez JR. Complex carbohydrates of rat tracheobronchial surface epithelium visualized ultrastructurally. Am J Anat 1980; 158: 93-109.

22 Lopez-Vidriero MT. Mucus as a natural barrier. Respiration 1989; 55: 28-32.

23 McDowell EM et al. The respiratory epithelium. I. Human bronchus. J Natl Cancer Inst 1978; 61: 539-549.

24 Kelm S, Schauer R. Sialic acids in molecular and cellular interactions. Int Rev Cytol 1997; 175: 137-240.

25 Roman J. Extracellular matrix and lung inflammation. Immunol Res 1996; 15: 163-178.

26 Madri JA, Furthmayr H. Isolation and tissue localization of type AB2 collagen from normal lung parenchyma. Am J Pathol 1979; 94: 323-331.

27 Aumailley M, Gayraud B. Structure and biological activity of the extracellular matrix. J Mol Med 1998; 76: 253-265.

28 Coyne CB, Kelly MM, Boucher RC, Johnson LG. Enhanced epithelial gene transfer by modulation of tight junctions with sodium caprate. Am J Respir Cell Mol Biol 2000; 23: 602-609.

29 Datema R, Schwarz RT. Interference with glycosylation of glycoproteins. Inhibition of formation of lipid-linked oligosaccharides in vivo. Biochem J 1979; 184: 113-123.

30 Gendler SJ, Spicer AP. Epithelial mucin genes. Annu Rev Physiol 1995; 57: 607-634.

31 Alberts B. Cell junctions, cell adhesion, and the extracellular matrix. In: Alberts B (ed.). Molecular Biology of the Cell. Garland Publishing: New York, 1994, pp 949-1009.

32 Dunsmore SE, Rannels DE. Extracellular matrix biology in the lung. Am J Physiol 1996; 270: L3-27.

33 Arcasoy SM et al. MUC1 and other sialoglycoconjugates inhibit adenovirus-mediated gene transfer to epithelial cells. Am J Respir Cell Mol Biol 1997; 17: 422-435.

34 Walters RW et al. Apical localization of the coxsackie-adenovirus receptor by glycosyl-phosphatidylinositol modification is sufficient for adenovirus-mediated gene transfer through the apical surface of human airway epithelia. J Virol 2001; 75: 7703-7711.

35 Goff SP. Intracellular trafficking of retroviral genomes during the early phase of infection: viral exploitation of cellular pathways. J Gene Med 2001; 3: 517-528.

36 Wang G et al. Human coronavirus 229E infects polarized airway epithelia from the apical surface. J Virol 2000; 74: 9234-9239.

37 Slepushkin VA et al. Infection of human airway epithelia with H1N1, H2N2 and H3N2 influenza A virus strains. Mol Ther 2001; 3: 395-402.

38 Yamaya M, Finkbeiner WE, Chun SY, Widdicombe JH. Differentiated structure and function of cultures from human tracheal epithelium. Am J Physiol 1992; 262: L713-L724.

39 Zabner J, Zeiher BG, Friedman E, Welsh MJ. Adenovirusmediated gene transfer to ciliated airway epithelia requires prolonged incubation time. J Virol 1996; 70: 6994-7003.

40 Anderson RD et al. A simple method for the rapid generation of recombinant adenovirus vectors. Gene Therapy 2000; 7: 1034-1038.

41 Sheridan PL et al. Generation of retroviral packaging and producer cell lines for large-scale vector production and clinical application: improved safety and high titer. Mol Ther 2000; 2: 262-275.

42 Cosset F-L et al. High-titer packaging cells producing recombinant retroviruses resistant to human serum. J Virol 1995; 69: 7430-7436.

43 Kitten O, Cosset F-L, Ferry N. Highly efficient retrovirusmediated gene transfer into rat hepatocytes in vivo. Hum Gene Ther 1997; 8: 1491-1494. 